\title{
Paraganglioma of the Larynx
}

\author{
Dhakhwa R ${ }^{1}$, KC Toran ${ }^{2}$, Kafle $\mathrm{S}^{1}$, Lakhey $\mathrm{M}^{1}$ \\ ${ }^{1}$ Department of Pathology, Kathmandu Medical College, Kathmandu, Nepal \\ ${ }^{2}$ Department of ENT and Head and Neck Surgery, Kathmandu Medical College, Kathmandu, Nepal
}

\section{Keywords:}

Immunohistochemistry;

Larynx, Neuroendocrine;

Paraganglioma

\begin{abstract}
Paraganglioma of the larynx is a rare tumor that arises from paraganglion cells. These tumors are generally considered benign and have to be differentiated from other neuroendocrine tumors. Diagnosis relies mostly on histopathologic examination followed by immunohistochemistry. Surgical excision generally confers complete cure of the tumor. We hereby present a case of laryngeal paraganglioma in a 60 -yearold female, who underwent total laryngectomy following a diagnostic micro-laryngeal biopsy, which was supported by immunohistochemistry.
\end{abstract}

\section{INTRODUCTION}

Paragangliomas are uncommon tumors that arise from the paraganglion system located throughout the body. ${ }^{1}$ Von Haller in 1743 was the first to describe paragangliomic tissue- the carotid body. Von Luschka in 1862 and Marchand in 1891 first described carotid body tumors. The nomenclature regarding paragangliomas has been confusing. Various authors have referred to these tumors as glomus tumors, chemodectomas, non chromaffin tumors and carotid body tumors. Glenner and Grimely in 1974 clarified this confusion by separating the tumors into adrenal paragangliomas or pheochromocytomas and extraadrenal tumors. ${ }^{2}$ Approximately $90 \%$ of tumors arising from the paraganglion system are in the adrenal gland. The remaining $10 \%$ arise from extra-adrenal sites with $85 \%$ arising in the abdomen, $12 \%$ in the thorax and the remaining $3 \%$ in the head and neck region. ${ }^{3}$ The carotid body is the most frequently reported site of paraganglioma in the head and neck region. Paragangliomas of the larynx are very rare tumors. They are a subclass of neuroendocrine tumors of the larynx with a neural origin. The other subclass

\section{Correspondence:}

Dr. Ramesh Dhakhwa, MD

Department of Pathology, Kathmandu Medical College, Kathmandu, Nepal E-mail:rdhakhwa@gmail.com of neuroendocrine tumors are of epithelial origin and include typical carcinoids, atypical carcinoids and small cell neuroendocrine carcinoma. ${ }^{4}$ Immunohistochemistry is helpful in distingusihing parangangliomas from other neuroendocrine tumors. ${ }^{5}$ Since its first description in the literature in 1955 , fewer than 80 cases of laryngeal paragangliomas have been reported. ${ }^{6}$ These benign lesions have a clinical course marked by slow growth, with symptoms often mimicking those of a squamous cell carcinoma. Patients present with hoarseness or dysphasia, and a submucosal mass is evident on laryngoscopic examination. The majority of these tumors arise in the supraglottic larynx, and $2 \%$ of these are malignant. ${ }^{7}$

\section{CASE REPORT}

A 60-year-old woman presented with hoarseness of voice and foreign body sensation in throat for five months. She was hypertensive with history of smoking and occasional alcohol intake. Fibreoptic laryngoscopy revealed a pink exophytic mass arising from epiglottis, right aryepiglottic fold and false cord. A provisional diagnosis of right supraglottic carcinoma was made and laryngoscopic biopsy was sent for histopathologic examination. Microscopic examination revealed submucosal tumor composed of atypical cells 


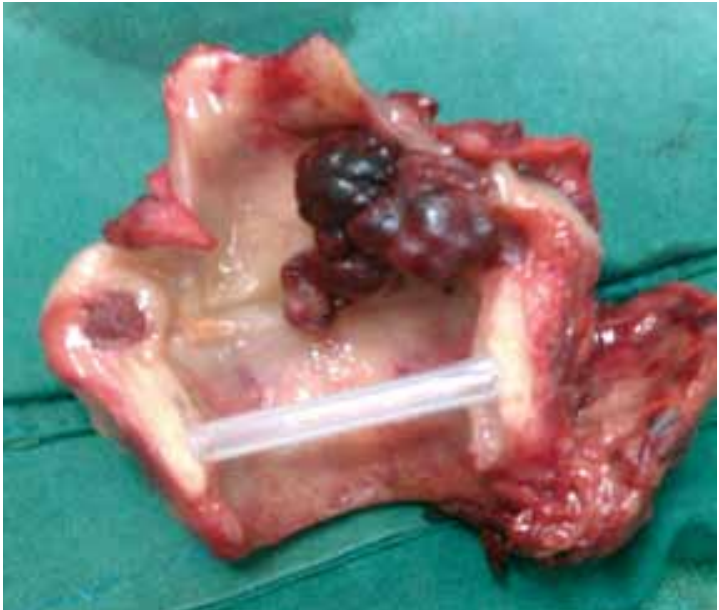

Figure 1: Laryngectomy specimen with tumor

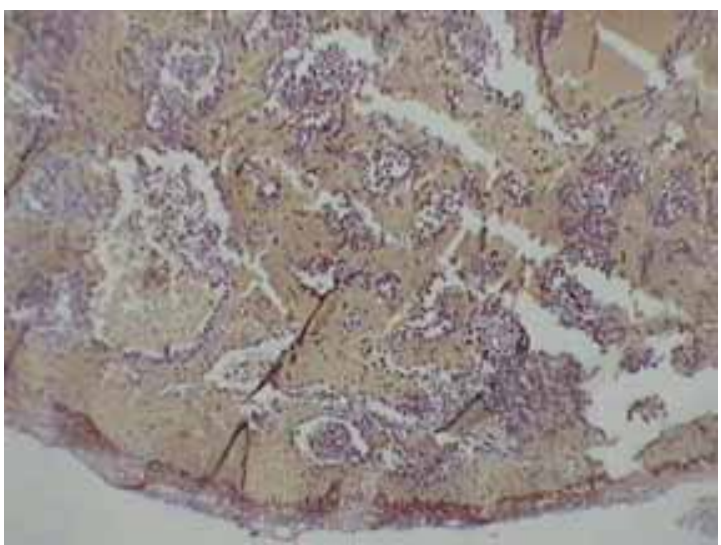

Figure 3A: Immunohistochemistry showing negative staining with Cytokeratin (X100).

arranged in nests and lobules separated by fibrovascular stroma. The tumor cells exhibited moderate pleomorphism with round to oval hyperchromatic nuclei. Cytoplasm was moderate to abundant, eosinophilic and granular. Congested capillaries and large areas of hemorrhage were noted. With these histopathologic findings, a provisional diagnosis of paraganglioma was made with a differential diagnosis of small cell variant of squamous cell carcinoma.

Subsequently, the patient underwent an elective total laryngectomy and the specimen was sent for histopathologic examination. Gross examination revealed a nodular grayish brown exophytic growth measuring $2.5 \times 2.5 \times 1 \mathrm{~cm}$, located in the right supraglottic region (fig. 1). Microscopic examination showed a subepithelial tumor composed of chief cells arranged in nests and characteristic zellballen pattern separated by fibrovascular stroma (fig. 2). The chief cells exhibited mild nuclear pleomorphism with round to oval nuclei and granular to fine chromatin. The sustentacular cells were located at the periphery of zellballen and had eosinophilic cytoplasm and angulated nuclei. Large areas of hemorrhage and congestion were noted. Lymphovascular and perineural invasion was not seen. The surgical margins

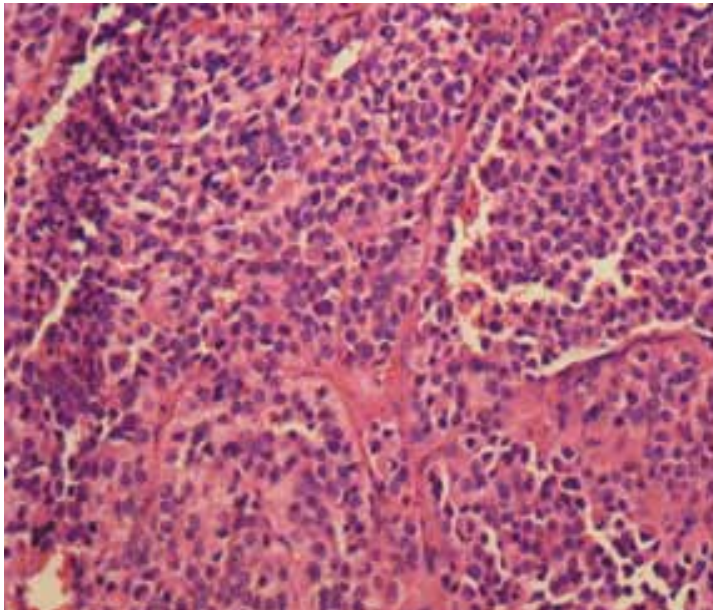

Figure 2: Light microscopy of excised tumor demonstrating Zellballen pattern (HE stain, X400).

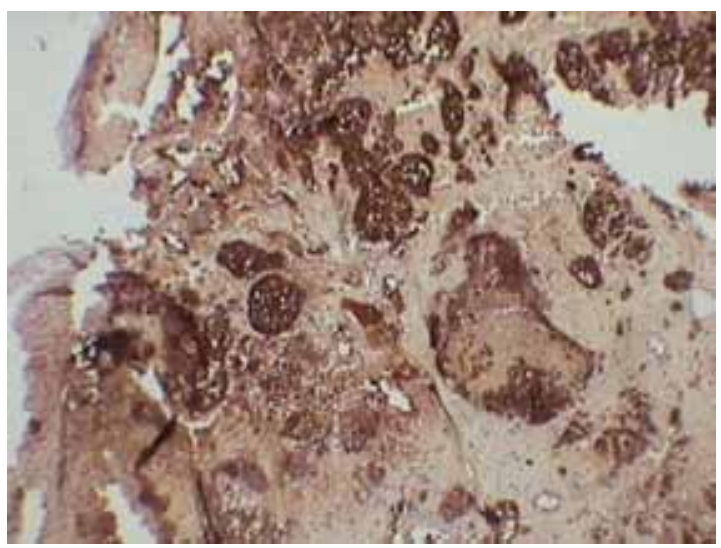

Figure 3B: Immnunohistochemistry showing positive staining with Chromogranin (X100).

were free of tumor. Immunohistochemistry for cytokeratin (fig. 3A) and chromogranin (fig. 3B) was done in the tissue section which revealed chromogranin positivity and cytokeratin negativity. A final diagnosis of paraganglioma was made.

\section{DISCUSSION}

The larynx contains two pairs of paraganglia that are divided into two groups: superior and inferior. The physiological role of laryngeal paraganglia is unknown. They may possibly serve as extracarotid chemoreceptors or have some effect on respiration via the larynx. ${ }^{8}$ Laryngeal paragangliomas are rare and are three times more common in women and have been described in patients from 5-83 years of age (median 44 years). The vast majority $(82 \%)$ occur in the supraglottic larynx, presumably arising from the superior pair of laryngeal paraganglia, and present as a submucosal mass in the region of the aryepiglottic fold - false vocal cord. Only $15 \%$ occur in the subglottis and $3 \%$ in the glottis. The right side of the larynx is more often involved than the left by a ratio $2.3: 1 .{ }^{6,9}$ 
Patients present with a wide variety of symptoms such as hoarseness, dysphagia, dyspnea, stridor, dysphonia, sore throat, hemoptysis etc. Multiple paragangliomas of the head and neck with laryngeal involvement have also been recorded. ${ }^{10}$

Microscopically, laryngeal paragangliomas have the same appearance as paragangliomas of other sites. The tumor is highly vascular and composed of two types of cells: chief cells and sustentacular cells, arranged in a characteristic alveolar or Zellballen pattern. The chief cells (type I cells, epithelioid cells) are more numerous and contain catecholamine bound neurosecretory granules as seen ultrastructurally. The sustentacular cells (type II cells, supporting cells) are devoid of neurosecretory granules and are characteristically located at the periphery of Zellballen. ${ }^{8}$ The "Zellballen" pattern is not diagnostic of a paragangioma as it may also exist in a variety of other tumors including typical and atypical carcinoid, malignant melanoma and medullary carcinoma of thyroid. Immunohistochemistry is helpful in establishing the correct diagnosis as the distinction between paraganglioma and atypical carcinoid/ neuroendocrine carcinoma may prove difficult on light microscopy. The presence of chromogranin positivity excludes non neuroendocrine neoplasms and the absence of keratin positivity and presence of S-100 protein-positive sustentacular cells tends to exclude carcinomas. ${ }^{11}$ In the present case, only a provisional diagnosis of paraganglioma could be made on histopathologic examination. However, with the help of immunohistochemistry (Chromogranin positive and Cytokeratin negative) a definitive diagnosis of paraganglioma was possible before surgical excision was performed.

Paragangliomas are generally benign tumors. The presence of vascular, capsular or perineural invasion does not necessarily indicate aggressive behavior. ${ }^{12}$ It is generally accepted that a paraganglioma is determined to be malignant only when metastasis is demonstrated. ${ }^{13}$ We consider the present case to be benign as there was no evidence of metastasis. The patient is however under regular follow-up to detect any recurrence/metastasis at an early stage.

\section{CONCLUSION}

Paraganglioma of larynx is rare. It has to be differentiated from other neuroendocrine tumors. Diagnosis is based on its characteristic histopathologic findings, supported by immunohistochemistry.

\section{REFERENCE}

1. Lack EE, Cubila AL, Woodruff JM, Farr HW. Paragangliomas of the head and neck region. A clinical study of 69 patients. Cancer 1977;39:397-409.

2. Myssiorek D. Head and Neck Paragangliomas, Otolaryngol Clinics N Am 2001;34:829-36.

3. Wasserman P, Savargaonkar P. Paragangliomas: Classification, Pathology, and Differential Diagnosis, Otolaryngol Clinics N Am 2002;34:845-62.

4. Gripp FM, Risse EKJ, Leverstein H, Snow GB, Meijer CJL. Neuroendocrine neoplasms of the larynx. Importance of the correct diagnosis and differences between atypical carcinoid tumours and small-cell neuroendocrine carcinoma. Eur Arch Otorhinolaryngol 1995;252:280-6.

5. Lack EE. Tumors of the autonomic nervous system (including paraganglia). In: Fletcher CDM, Diagnostic Histopathology of Tumors, 3rd Edn, Churchill Livingstone 2007. pp 1768.

6. Ferito A, Barnes L, Wenig BM: Identification, classification, treatment, and prognosis of laryngeal paraganglioma. Review of the literature and eight new cases. Ann Otol Rhinol Laryngol 1994;103:525-36.

7. Smolarz JR, Hanna EY, Williams MD and Kupferman ME: Paraganglioma of the endolarynx: a rare tumor in an uncommon location. Head Neck Oncol 2010;2:2.

8. Barnes L, Tse LLY, Hunt JL, Michaels L. Tumors of Paraganglionic system: Introduction. In: Barnes L, Eveson JW, Reichart P, Sidransky D, Editors. Pathology and Genetics. Head and Neck Tumors, Lyon: IARC Press; 2005; pp 362-4.

9. Barnes L. Paraganglioma of the larynx. A critical review of the literature. ORL J Otorhinolaryngol Relat Spec 1991;53:220-34.

10. Khademi B, Taghizadegan L, Gandomi B, Vasei M: Paraganglioma of the larynx: A case report. IJMS 2004;29:47-9.

11. Pilch BZ. Larynx and trachea; Benign neoplasms. In: Fletcher CDM, Diagnostic Histopathology of Tumors, 3rd Edn. Churchill Livingstone 2007.pp153.

12. Ferlito A, Milory CM, Wenig BM et al. Laryngeal paraganglioma versus atypical carcinoid tumor. Ann Otol Rhinol Laryngol 1995;104:78-83.

13. Kimura N, Chetty R, Capella $\mathrm{C}$ et al. Extra-adrenal paraganglioma: Carotid body, jugulotympanic, vagal, laryngeal, aortico-pulmonary. In: DeLellis, Lloyd RV, Heitz PU, Eng C, Editors. Pathology and Genetics. Tumors of Endocrine Organs, Lyon: IARC Press, 2004. $16 \mathrm{pp}$. 